\title{
Cauchy-Bunyakovsky-Schwarz type inequalities related to Möbius operations
}

Keiichi Watanabe 1* (D) $^{*}$

Dedicated to Professor Osamu Hatori on his 65th birthday

"Correspondence:

wtnbk@math.sc.niigata-u.ac.jp ${ }^{1}$ Department of Mathematics,

Faculty of Science, Niigata

University, Niigata, Japan

\begin{abstract}
We show some Cauchy-Bunyakovsky-Schwarz type inequalities related to the Möbius operations in complex inner product spaces which are extensions of a similar type inequality obtained recently, and we discuss some properties.

MSC: Primary 46C05; secondary 20N05; 26D20; 46T99; 51M10; 83A05

Keywords: Schwarz inequality; Möbius addition; Möbius scalar multiplication; Möbius gyrovector space
\end{abstract}

\section{Introduction and preliminaries}

The celebrated Cauchy-Bunyakovsky-Schwarz (CBS in the sequel) inequality

$$
|\langle u, v\rangle| \leq\langle u, u\rangle^{\frac{1}{2}}\langle v, v\rangle^{\frac{1}{2}} \quad(u, v \in \mathbb{V})
$$

in an arbitrary inner product space $\mathbb{V}$ is one of the most fundamental inequalities in mathematics.

Möbius addition is defined on the complex open unit disk $\mathbb{D}=\{z \in \mathbb{C} ;|z|<1\}$ by

$$
a \oplus b=\frac{a+b}{1+\bar{a} b} \quad(a, b \in \mathbb{D}),
$$

which appears in a wide variety of fields of mathematics. In particular, although Möbius addition is known in the literature as hyperbolic translation, its group-like structure had gone unnoticed until it was uncovered by A.A. Ungar in 1988 [4], in the context of Einstein's special theory of relativity. Furthermore, Ungar extended Möbius addition, introduced Möbius scalar multiplication to open balls of arbitrary real inner product spaces, and established the concept of gyrovector spaces, which have a vector space-like structure (see [4-6]).

Let us briefly recall the definition of the Möbius gyrovector spaces. For precise definitions and basic results of gyrocommutative gyrogroups and gyrovector spaces, see [7]. Although one does not need any knowledge on gyrovector space theory to read this paper, it is a significant portion of our motivation and background. In addition, notations from gyrovector space theory simplify expressions of our inequalities remarkably. For elementary facts on inner product spaces and CBS type inequalities, one can refer to $[1,3]$.

(c) The Author(s) 2019. This article is distributed under the terms of the Creative Commons Attribution 4.0 International License (http://creativecommons.org/licenses/by/4.0/), which permits unrestricted use, distribution, and reproduction in any medium, provided you give appropriate credit to the original author(s) and the source, provide a link to the Creative Commons license, and indicate if changes were made. 
Definition 1.1 ([7, Definition 3.40 and 6.83]) Let $\mathbb{V}=(\mathbb{V},+,\langle\cdot, \cdot\rangle)$ be a real inner product space with a vector addition + and a positive definite inner product $\langle\cdot, \cdot\rangle$. Let $\mathbb{V}_{s}$ be an open ball

$$
\mathbb{V}_{s}=\{v \in \mathbb{V} ;\|v\|<s\}
$$

for any fixed $s>0$, where $\|v\|=\langle v, v\rangle^{\frac{1}{2}}$. The Möbius addition $\oplus_{M}$ and the Möbius scalar multiplication $\otimes_{\mathrm{M}}$ are given by the equations

$$
\begin{aligned}
& u \oplus_{M} v=\frac{\left(1+\frac{2}{s^{2}}\langle u, v\rangle+\frac{1}{s^{2}}\|v\|^{2}\right) u+\left(1-\frac{1}{s^{2}}\|u\|^{2}\right) v}{1+\frac{2}{s^{2}}\langle u, v\rangle+\frac{1}{s^{4}}\|u\|^{2}\|v\|^{2}}, \\
& r \otimes_{\mathrm{M}} u=s \tanh \left(r \tanh ^{-1} \frac{\|u\|}{s}\right) \frac{u}{\|u\|} \quad(\text { if } u \neq 0), r \otimes_{\mathrm{M}} 0=0,
\end{aligned}
$$

for any $u, v \in \mathbb{V}_{s}$ and $r \in \mathbb{R}$. The addition $\oplus_{M}$ and the scalar multiplication $\otimes_{M}$ for real numbers are defined by the equations

$$
\begin{aligned}
& a \oplus_{\mathrm{M}} b=\frac{a+b}{1+\frac{1}{s^{2}} a b}, \\
& r \otimes_{\mathrm{M}} a=s \tanh \left(r \tanh ^{-1} \frac{a}{s}\right)
\end{aligned}
$$

for any $a, b \in(-s, s)$ and $r \in \mathbb{R}$.

We simply denote $\oplus_{M}, \otimes_{M}$ by $\oplus_{s}, \otimes_{s}$, respectively.

Theorem 1.2 (see [7, after Remark 3.41], [6, p. 1054]) The Möbius addition (resp. Möbius scalar multiplication) reduces to the ordinary vector addition (resp. scalar multiplication) as $s \rightarrow \infty$, that is,

$$
\begin{aligned}
& u \oplus_{s} v \rightarrow u+v \quad(s \rightarrow \infty), \\
& r \otimes_{s} u \rightarrow r u \quad(s \rightarrow \infty)
\end{aligned}
$$

Definition 1.3 ([7, Definition 2.7, (2.1), (6.286), (6.293)]) The inverse element of $u$ with respect to $\oplus_{s}$ obviously coincides with $-u$. We use the notation

$$
u \ominus_{s} v=u \oplus_{s}(-v)
$$

as in group theory. Moreover, the Möbius gyrodistance function $d$ and Poincaré distance function $h$ are defined by the equations

$$
\begin{aligned}
& d(u, v)=\left\|v \ominus_{s} u\right\|, \\
& h(u, v)=\tanh ^{-1} \frac{d(u, v)}{s} .
\end{aligned}
$$

Ungar showed that $h$ satisfies the triangle inequality [7, (6.294)]. 
The following identities are an easy consequence of the definition. One can refer to [10, Lemma 14 (i), Lemma 12].

Lemma 1.4 Let $s>0$. The following formulae hold:

(i) $\frac{u}{s} \oplus_{1} \frac{v}{s}=\frac{u \oplus_{s} v}{s}$,

(ii) $\left\|u \oplus_{s} v\right\|^{2}=\frac{\|u\|^{2}+2\langle u, v\rangle+\|v\|^{2}}{1+\frac{2}{s^{2}}\langle u, v\rangle+\frac{1}{s^{4}}\|u\|^{2}\|v\|^{2}}$

for any $u, v \in \mathbb{V}_{s}$.

Note that the Möbius operations generally are not commutative, associative, or distributive. Furthermore, the ordinary scalar multiplication does not distribute the Möbius addition. However, the restricted Möbius operations to real numbers together with the ordinary addition and multiplication have the following properties:

$$
\begin{aligned}
& \left(r_{1} r_{2}\right) \otimes_{s} a=r_{1} \otimes_{s}\left(r_{2} \otimes_{s} a\right), \\
& \left(r_{1}+r_{2}\right) \otimes_{s} a=r_{1} \otimes_{s} a \oplus_{s} r_{2} \otimes_{s} a, \\
& r \otimes_{s}\left(a \oplus_{s} b\right)=r \otimes_{s} a \oplus_{s} r \otimes_{s} b
\end{aligned}
$$

for any $-s<a, b<s, r_{1}, r_{2}, r \in \mathbb{R}$. It follows from the additive formula of the hyperbolic function. In addition, we need the following lemmas.

Lemma 1.5 If $0 \leq b_{j} \leq a_{j}<s(j=1,2)$, then

$$
b_{1} \oplus_{s} b_{2} \leq a_{1} \oplus_{s} a_{2} .
$$

The equality holds if and only if $a_{j}=b_{j}(j=1,2)$.

Proof We may assume $s=1$. It is immediate to see that

$$
\begin{aligned}
& \left(a_{1}+a_{2}\right)\left(1+b_{1} b_{2}\right)-\left(1+a_{1} a_{2}\right)\left(b_{1}+b_{2}\right) \\
& \quad=\left(a_{1}-b_{1}\right)\left(1-a_{2} b_{2}\right)+\left(a_{2}-b_{2}\right)\left(1-a_{1} b_{1}\right) \geq 0,
\end{aligned}
$$

which implies

$$
\frac{b_{1}+b_{2}}{1+b_{1} b_{2}} \leq \frac{a_{1}+a_{2}}{1+a_{1} a_{2}} .
$$

The equality condition also follows from the proof above. This completes the proof.

Lemma 1.6 If $0<a<s$ and $0<r<1$ (resp. $r>1)$, then $r a<r \otimes_{s} a\left(\right.$ resp. $\left.r a>r \otimes_{s} a\right)$.

Proof By the identity

$$
r \otimes_{1} \frac{a}{s}=\frac{r \otimes_{s} a}{s},
$$


we may assume $s=1$. Obviously, $r a<r \otimes_{1} a$ is equivalent to $\tanh ^{-1}(r a)<r \tanh ^{-1} a$. Considering $a$ as a variable, we put $f(x)=r \tanh ^{-1} x-\tanh ^{-1}(r x)$. Then it is easy to see

$$
f^{\prime}(x)=\frac{r\left(1-r^{2}\right) x^{2}}{\left(1-x^{2}\right)\left(1-r^{2} x^{2}\right)}>0 \quad(0<x<1)
$$

which yields the result for $0<r<1$. It is also easy to see the assertion for $r>1$. This completes the proof.

Recently, the following CBS type inequality related to the Möbius addition in complex inner product spaces was obtained. See also [8] for a discrete Cauchy type inequality including a mixture of the Möbius addition and the Möbius scalar multiplication restricted to real numbers.

Theorem 1.7 ([9, Theorem 5]) Let $\mathbb{V}$ be a complex inner product space, and let $w \in \mathbb{V}$ be a fixed element with $\|w\| \leq 1$. For any $u, v \in \mathbb{V}$ and for any $s>\max \{\|u\|,\|v\|\}$, the following inequality holds:

$$
\left|\frac{\langle u, w\rangle-\langle v, w\rangle}{1-\frac{1}{s^{2}} \overline{\langle u, w\rangle}\langle v, w\rangle}\right| \leq \sqrt{\frac{\|u\|^{2}-2 \operatorname{Re}\langle u, v\rangle+\|v\|^{2}}{1-\frac{2}{s^{2}} \operatorname{Re}\langle u, v\rangle+\frac{1}{s^{4}}\|u\|^{2}\|v\|^{2}}} .
$$

The equality holds if and only if one of the following conditions is satisfied:

(i) $u=v$;

(ii) $\|w\|=1$ and $u=\lambda w, v=\mu w$ for some complex numbers $\lambda$, $\mu$.

Remark 1.8 As described in [9, Theorem 8], the corresponding inequality

$$
\left|\frac{\langle u, w\rangle-\langle v, w\rangle}{1-\frac{1}{s^{2}}\langle u, w\rangle\langle v, w\rangle}\right| \leq \sqrt{\frac{\|u\|^{2}-2\langle u, v\rangle+\|v\|^{2}}{1-\frac{2}{s^{2}}\langle u, v\rangle+\frac{1}{s^{4}}\|u\|^{2}\|v\|^{2}}}
$$

holds in real inner product spaces, and it can be simply expressed as

$$
\left|\langle u, w\rangle \ominus_{s}\langle v, w\rangle\right| \leq\left\|u \ominus_{s} v\right\|
$$

by using the Möbius subtraction. Note that generally $\langle u, w\rangle \ominus_{s}\langle v, w\rangle \neq\left\langle u \ominus_{s} v, w\right\rangle$.

Let us briefly compare the classical CBS inequality and inequality (1.1), and consider their relation. For any elements $u, v, w \in \mathbb{V}$, replacing $w$ by $\frac{w}{\|w\|}$ in inequality (1.1) and letting $s \rightarrow \infty$, then it asymptotically goes to the classical CBS inequality

$$
|\langle u, w\rangle-\langle v, w\rangle| \leq\|u-v\|\|w\|
$$

Clearly $\|w\|$ appears in the right-hand side of inequality (1.2). On the other hand, inequality (1.1) is not the case, even though it must be taken into account that the assumption of Theorem 1.7 includes the condition $\|w\| \leq 1$.

Example 1.9 In the one-dimensional complex inner product space $\mathbb{C}$, let

$$
u=\frac{1}{\sqrt{2}}, \quad v=-\frac{1}{\sqrt{2}}, \quad w=\frac{1}{\sqrt{2}} .
$$


Obviously $\|u\|,\|v\|<1$ and $\|w\| \leq 1$, and it is immediate to see

$$
\left|\frac{\langle u, w\rangle-\langle v, w\rangle}{1-\overline{\langle u, w\rangle}\langle v, w\rangle}\right|=\frac{4}{5}>\frac{2}{3}=\sqrt{\frac{\|u\|^{2}-2 \operatorname{Re}\langle u, v\rangle+\|v\|^{2}}{1-2 \operatorname{Re}\langle u, v\rangle+\|u\|^{2}\|v\|^{2}}}\|w\| .
$$

Despite the previous example, some kind of similar inequality involving $\|w\|$ in the righthand side may still be possible. Actually, S.-E. Takahasi raised the following question at a conference when Theorem 1.7 was presented.

Question 1.10 Is there any constant $C>1$ satisfying

$$
\left|\frac{\langle u, w\rangle-\langle v, w\rangle}{1-\overline{\langle u, w\rangle}\langle v, w\rangle}\right| \leq C \sqrt{\frac{\|u\|^{2}-2 \operatorname{Re}\langle u, v\rangle+\|v\|^{2}}{1-2 \operatorname{Re}\langle u, v\rangle+\|u\|^{2}\|v\|^{2}}}\|w\|
$$

for any $u, v, w \in \mathbb{V}$ with $\|u\|,\|v\|<1$ and $\|w\| \leq 1$ ?

In this article, we show some further extensions of CBS type inequality (1.1). One of them gives an affirmative and even sharper answer to Question 1.10. Another extension is related to both the Möbius addition and Möbius scalar multiplication, which can be regarded as the most natural extension in the context of relation between inner product spaces and the Möbius operations.

\section{An extension of the CBS type inequality}

In this section, we prove an extension of Theorem 1.7 related to the Möbius addition and discuss some properties. The following lemma is due to one of the referees.

Lemma 2.1 Let a be a real number and $z$ be a complex number. If $|z|<a<1$, then $\frac{1+a}{2 a}<$ $\left|\frac{1-z}{a-z}\right|$.

Proof It is enough to show that $4 a^{2}|1-z|^{2}-(1+a)^{2}|a-z|^{2}>0$. We can easily calculate

$$
\begin{aligned}
& 4 a^{2}|1-z|^{2}-(1+a)^{2}|a-z|^{2} \\
& \quad=3 a^{2}-2 a^{3}-a^{4}+2 a(1-a)^{2} \operatorname{Re} z-|z|^{2}(1-a)(3 a+1) \\
& >3 a^{2}-2 a^{3}-a^{4}-2 a^{2}(1-a)^{2}-a^{2}(1-a)(3 a+1) \\
& \quad=0 .
\end{aligned}
$$

This completes the proof.

The following theorem is an extension of CBS type inequality in complex inner product spaces related to the Möbius addition.

Theorem 2.2 Let $\mathbb{V}$ be a complex inner product space. For any $u, v \in \mathbb{V}, s>\max \{\|u\|,\|v\|\}$ and $w \in \mathbb{V}$ with $\|w\| \leq 1$, the following inequality holds:

$$
\left|\frac{\langle u, w\rangle-\langle v, w\rangle}{1-\frac{1}{s^{2}} \overline{\langle u, w\rangle}\langle v, w\rangle}\right| \leq \sqrt{\frac{\|u\|^{2}-2 \operatorname{Re}\langle u, v\rangle+\|v\|^{2}}{1-\frac{2}{s^{2}} \operatorname{Re}\langle u, v\rangle+\frac{1}{s^{4}}\|u\|^{2}\|v\|^{2}}} \cdot \frac{2\|w\|}{1+\|w\|^{2}} .
$$


The equality holds if and only if one of the following conditions is satisfied:

(i) $u=v$,

(ii) $w=0$,

(iii) $\|w\|=1$ and $u=\lambda w, v=\mu w$ for some complex numbers $\lambda, \mu$.

The author had given a proof of Theorem 2.2 in the original manuscript, whose method was quite similar to that in [9]. One of the referees pointed out that Theorem 2.2 can be easily deduced from Lemma 2.1 and Theorem 1.7, and kindly presented the proof below, which is remarkably shorter than the original one.

Proof It is easy to see that we may assume $s=1$. If $\|w\|=1$, then inequality (2.1) just reduces to inequality (1.1), so we may assume $0<\|w\|<1$ as well. Put

$$
a=\|w\|^{2}, \quad Y=\langle u, w\rangle-\langle v, w\rangle, \quad Z=\overline{\langle u, w\rangle}\langle v, w\rangle .
$$

Then the classical CBS inequality shows that $|Z| \leq\|u\|\|v\|\|w\|^{2}<a$. It follows from Lemma 2.1 that

$$
\frac{1+a}{2 \sqrt{a}}\left|\frac{Y}{1-Z}\right| \leq \sqrt{a}\left|\frac{1-Z}{a-Z}\right|\left|\frac{Y}{1-Z}\right|=\left|\frac{\sqrt{a} Y}{a-Z}\right|=\left|\frac{\frac{Y}{\sqrt{a}}}{1-\frac{Z}{a}}\right|,
$$

where the equality holds if and only if $Y=0$. This shows that

$$
\begin{aligned}
\frac{1+\|w\|^{2}}{2\|w\|}\left|\frac{\langle u, w\rangle-\langle v, w\rangle}{1-\overline{\langle u, w\rangle}\langle v, w\rangle}\right| & \leq\left|\frac{\left\langle u, \frac{w}{\|w\|}\right\rangle-\left\langle v, \frac{w}{\|w\|}\right\rangle}{\left.\left.1-\overline{\left\langle u, \frac{w}{\|w\|}\right\rangle}\right\rangle v, \frac{w}{\|w\|}\right\rangle}\right| \\
& \leq \sqrt{\frac{\|u\|^{2}-2 \operatorname{Re}\langle u, v\rangle+\|v\|^{2}}{1-2 \operatorname{Re}\langle u, v\rangle+\|u\|^{2}\|v\|^{2}}},
\end{aligned}
$$

where we used Theorem 1.7 for the later inequality. Thus we obtain the desired inequality. Next, we show the equality condition.

It is immediate to see that the equality in (2.1) holds provided one of the conditions (i), (ii), or (iii) is satisfied.

Conversely, suppose that the equality in (2.1) holds and $s=1$. It is obvious that we may assume $0<\|w\|<1$. Then, by the equality in the second inequality of (2.2) and the equality condition of Theorem 1.7, we have $u=v$ or $u=\lambda w, v=\mu w$ for some complex numbers $\lambda$, $\mu$. In the later case, the equality in the first inequality of (2.2) yields

$$
\lambda\|w\|^{2}=\langle\lambda w, w\rangle=\langle u, w\rangle=\langle v, w\rangle=\langle\mu w, w\rangle=\mu\|w\|^{2},
$$

so we obtain $u=v$. This completes the proof.

Remark 2.3 Since $\frac{2\|w\|}{1+\|w\|^{2}} \leq 1$, Theorem 2.2 is an improvement of Theorem 1.7.

The following corollary gives an affirmative answer to Question 1.10 with $C=2$. 
Corollary Let $\mathbb{V}$ be a complex inner product space. For any $u, v \in \mathbb{V}, s>\max \{\|u\|,\|v\|\}$ and $w \in \mathbb{V}$ with $\|w\| \leq 1$, the following inequality holds:

$$
\left|\frac{\langle u, w\rangle-\langle v, w\rangle}{1-\frac{1}{s^{2}} \overline{\langle u, w\rangle}\langle v, w\rangle}\right| \leq 2 \sqrt{\frac{\|u\|^{2}-2 \operatorname{Re}\langle u, v\rangle+\|v\|^{2}}{1-\frac{2}{s^{2}} \operatorname{Re}\langle u, v\rangle+\frac{1}{s^{4}}\|u\|^{2}\|v\|^{2}}}\|w\| .
$$

The following theorem shows that the constant 2 in the right-hand side of inequalities (2.1) and (2.3) is the best in a sense.

Theorem 2.4 For any real number $C<2$, there exist elements $u, v, w \in \mathbb{V}$ satisfying $\|u\|,\|v\|<1,\|w\| \leq 1$ and

$$
\left|\frac{\langle u, w\rangle-\langle v, w\rangle}{1-\overline{\langle u, w\rangle}\langle v, w\rangle}\right|>C \sqrt{\frac{\|u\|^{2}-2 \operatorname{Re}\langle u, v\rangle+\|v\|^{2}}{1-2 \operatorname{Re}\langle u, v\rangle+\|u\|^{2}\|v\|^{2}}}\|w\| .
$$

Proof In the one-dimensional complex inner product space $\mathbb{C}$, let

$$
u_{n}=\sqrt{1-\frac{1}{n}}, \quad v_{n}=-\sqrt{1-\frac{1}{n}}, \quad w_{n}=\frac{1}{\sqrt{n}} .
$$

Obviously $\left\|u_{n}\right\|,\left\|v_{n}\right\|<1$ and $\left\|w_{n}\right\| \leq 1$, and it is immediate to see

$$
\begin{aligned}
& \left|\frac{\left\langle u_{n}, w_{n}\right\rangle-\left\langle v_{n}, w_{n}\right\rangle}{1-\overline{\left\langle u_{n}, w_{n}\right\rangle}\left\langle v_{n}, w_{n}\right\rangle}\right|=\frac{2 n \sqrt{n-1}}{n^{2}+n-1}, \\
& \sqrt{\frac{\left\|u_{n}\right\|^{2}-2 \operatorname{Re}\left\langle u_{n}, v_{n}\right\rangle+\left\|v_{n}\right\|^{2}}{1-2 \operatorname{Re}\left\langle u_{n}, v_{n}\right\rangle+\left\|u_{n}\right\|^{2}\left\|v_{n}\right\|^{2}}}=\frac{2 \sqrt{n(n-1)}}{2 n-1} .
\end{aligned}
$$

Therefore we have

$$
\begin{aligned}
& \left|\frac{\left\langle u_{n}, w_{n}\right\rangle-\left\langle v_{n}, w_{n}\right\rangle}{1-\overline{\left\langle u_{n}, w_{n}\right\rangle}\left\langle v_{n}, w_{n}\right\rangle}\right| / \sqrt{\frac{\left\|u_{n}\right\|^{2}-2 \operatorname{Re}\left\langle u_{n}, v_{n}\right\rangle+\left\|v_{n}\right\|^{2}}{1-2 \operatorname{Re}\left\langle u_{n}, v_{n}\right\rangle+\left\|u_{n}\right\|^{2}\left\|v_{n}\right\|^{2}}}\left\|w_{n}\right\| \\
& =\frac{n(2 n-1)}{n^{2}+n-1} \rightarrow 2(n \rightarrow \infty) .
\end{aligned}
$$

This completes the proof.

If the following inequality

$$
\left|\frac{1}{1-\overline{\langle u, w\rangle}\langle v, w\rangle}\right| \leq \sqrt{\frac{1}{1-2 \operatorname{Re}\langle u, v\rangle+\|u\|^{2}\|v\|^{2}}} \cdot \frac{2}{1+\|w\|^{2}}
$$

would be valid for any $u, v, w \in \mathbb{V}$ with $\|u\|,\|v\|<1,\|w\| \leq 1$, then we could prove Theorem 2.2 simply by combining the classical CBS inequality (1.2) and inequality (2.4). However, this is not the case. 
Theorem 2.5 For any real number $C>0$, there exist elements $u, v, w \in \mathbb{V}$ satisfying $\|u\|,\|v\|<1,\|w\| \leq 1$, and

$$
\left|\frac{1}{1-\overline{\langle u, w\rangle}\langle v, w\rangle}\right|>C \sqrt{\frac{1}{1-2 \operatorname{Re}\langle u, v\rangle+\|u\|^{2}\|v\|^{2}}} \cdot \frac{2}{1+\|w\|^{2}} .
$$

Proof Take a real number $0<a<1$ satisfying $\frac{1+3 a}{(1-a)(1+a)^{2}}>C^{2}$. In the two-dimensional complex inner product space $\mathbb{C}^{2}$, let

$$
u=\left(\begin{array}{c}
a \\
\sqrt{a(1-a)}
\end{array}\right), \quad v=\left(\begin{array}{c}
a \\
-\sqrt{a(1-a)}
\end{array}\right), \quad w=\left(\begin{array}{l}
1 \\
0
\end{array}\right) .
$$

Obviously $\|u\|,\|v\|<1$ and $\|w\|=1$, and it is immediate to see

$$
\begin{aligned}
& |1-\overline{\langle u, w\rangle}\langle v, w\rangle|=1-a^{2}, \\
& 1-2 \operatorname{Re}\langle u, v\rangle+\|u\|^{2}\|v\|^{2}=1+2 a-3 a^{2} .
\end{aligned}
$$

Since $1+2 a-3 a^{2}>C^{2}\left(1-a^{2}\right)^{2}$, we obtain

$$
1-2 \operatorname{Re}\langle u, v\rangle+\|u\|^{2}\|v\|^{2}>C^{2}|1-\overline{\langle u, w\rangle}\langle v, w\rangle|^{2} .
$$

This completes the proof.

Remark 2.6 One can easily modify the proof of Theorem 2.2 to obtain the corresponding inequality in real inner product spaces.

Theorem 2.7 Let $\mathbb{V}$ be a real inner product space. For any $u, v \in \mathbb{V}, s>\max \{\|u\|,\|v\|\}$ and $w \in \mathbb{V}$ with $\|w\| \leq 1$, the following inequality holds:

$$
\left|\frac{\langle u, w\rangle-\langle v, w\rangle}{1-\frac{1}{s^{2}}\langle u, w\rangle\langle v, w\rangle}\right| \leq \sqrt{\frac{\|u\|^{2}-2\langle u, v\rangle+\|v\|^{2}}{1-\frac{2}{s^{2}}\langle u, v\rangle+\frac{1}{s^{4}}\|u\|^{2}\|v\|^{2}}} \cdot \frac{2\|w\|}{1+\|w\|^{2}} .
$$

The equality holds if and only if one of the following conditions is satisfied:

(i) $u=v$,

(ii) $w=0$,

(iii) $\|w\|=1$ and $u=\lambda w, v=\mu w$ for some real numbers $\lambda, \mu$.

\section{A CBS type inequality related to the Möbius operations}

In this section, we prove a rather essential extension of Theorem 1.7, which is related to both the Möbius addition and Möbius scalar multiplication.

The next is a key result for the main theorem, which might be interesting by itself as an elementary inequality of real and complex numbers. 
Theorem 3.1 Let $p$ and $q$ be real numbers with $0 \leq p, q \leq 1$, and let $\alpha$ and $\beta$ be complex numbers with $|\alpha|,|\beta| \leq 1$. Then the following inequality holds:

$$
\begin{aligned}
& (p+q)\left|1-\frac{(p+q)^{2}}{4} \bar{\alpha} \beta\right|\left\{\left|1-p^{2} \bar{\alpha} \beta\right|\left|1-q^{2} \bar{\alpha} \beta\right|+p q|\alpha-\beta|^{2}\right\} \\
& \quad \leq\left\{p\left|1-q^{2} \bar{\alpha} \beta\right|+q\left|1-p^{2} \bar{\alpha} \beta\right|\right\}\left\{\left|1-\frac{(p+q)^{2}}{4} \bar{\alpha} \beta\right|^{2}+\frac{(p+q)^{2}}{4}|\alpha-\beta|^{2}\right\} .
\end{aligned}
$$

The equality holds if and only if one of the following conditions is satisfied:

(i) $p=q$,

(ii) $\alpha=\beta=0$.

Proof We will give a proof of Theorem 3.1 by showing each proof of a number of lemmas. Let $\alpha=a e^{i x}, \beta=b e^{i y}$ with $0 \leq a, b \leq 1$ and $0 \leq x, y \leq 2 \pi$ be the polar form of complex numbers $\alpha, \beta$. Put $t=\cos (-x+y)$ and

$$
\begin{aligned}
& A=\left|1-p^{2} \bar{\alpha} \beta\right|=\sqrt{1-2 p^{2} a b t+p^{4} a^{2} b^{2}}, \\
& B=\left|1-q^{2} \bar{\alpha} \beta\right|=\sqrt{1-2 q^{2} a b t+q^{4} a^{2} b^{2}}, \\
& C=\left|1-\frac{(p+q)^{2}}{4} \bar{\alpha} \beta\right|=\sqrt{1-\frac{1}{2}(p+q)^{2} a b t+\frac{1}{16}(p+q)^{4} a^{2} b^{2}}, \\
& D=|\alpha-\beta|=\sqrt{a^{2}-2 a b t+b^{2}} .
\end{aligned}
$$

We will show

$$
(p+q)^{2} C^{2}\left\{A B+p q D^{2}\right\}^{2} \leq\{p B+q A\}^{2}\left\{C^{2}+\frac{(p+q)^{2}}{4} D^{2}\right\}^{2},
$$

because it seems difficult to prove directly

$$
(p+q) C\left\{A B+p q D^{2}\right\} \leq\{p B+q A\}\left\{C^{2}+\frac{(p+q)^{2}}{4} D^{2}\right\} .
$$

We can calculate the subtraction of the left-hand side from the right-hand side of (3.1) as follows:

$$
\begin{aligned}
\{p B+ & q A\}^{2}\left\{C^{2}+\frac{(p+q)^{2}}{4} D^{2}\right\}^{2}-(p+q)^{2} C^{2}\left\{A B+p q D^{2}\right\}^{2} \\
= & \left\{p^{2} B^{2}+q^{2} A^{2}\right\}\left\{C^{4}+\frac{1}{2}(p+q)^{2} C^{2} D^{2}+\frac{1}{16}(p+q)^{4} D^{4}\right\} \\
& -(p+q)^{2} C^{2}\left\{A^{2} B^{2}+p^{2} q^{2} D^{4}\right\} \\
& +2 p q A B\left\{C^{4}-\frac{1}{2}(p+q)^{2} C^{2} D^{2}+\frac{1}{16}(p+q)^{4} D^{4}\right\} .
\end{aligned}
$$

Lemma 3.2 The sum of the first two terms

$$
\left\{p^{2} B^{2}+q^{2} A^{2}\right\}\left\{C^{4}+\frac{1}{2}(p+q)^{2} C^{2} D^{2}+\frac{1}{16}(p+q)^{4} D^{4}\right\}-(p+q)^{2} C^{2}\left\{A^{2} B^{2}+p^{2} q^{2} D^{4}\right\}
$$

in formula (3.3) is a polynomial whose degree is at most 1 with respect to $t$. 
Proof

$$
\begin{aligned}
\left\{p^{2} B^{2}\right. & \left.+q^{2} A^{2}\right\}\left\{C^{4}+\frac{1}{2}(p+q)^{2} C^{2} D^{2}+\frac{1}{16}(p+q)^{4} D^{4}\right\} \\
& -(p+q)^{2} C^{2}\left\{A^{2} B^{2}+p^{2} q^{2} D^{4}\right\} \\
= & \left\{p^{2}\left(1-2 q^{2} a b t+q^{4} a^{2} b^{2}\right)+q^{2}\left(1-2 p^{2} a b t+p^{4} a^{2} b^{2}\right)\right\} \\
& \times\left\{1-\frac{1}{2}(p+q)^{2} a b t+\frac{1}{16}(p+q)^{4} a^{2} b^{2}\right\}^{2} \\
& +\frac{1}{2}(p+q)^{2}\left\{p^{2}\left(1-2 q^{2} a b t+q^{4} a^{2} b^{2}\right)+q^{2}\left(1-2 p^{2} a b t+p^{4} a^{2} b^{2}\right)\right\} \\
& \times\left\{1-\frac{1}{2}(p+q)^{2} a b t+\frac{1}{16}(p+q)^{4} a^{2} b^{2}\right\}\left(a^{2}-2 a b t+b^{2}\right) \\
& +\frac{1}{16}(p+q)^{4}\left\{p^{2}\left(1-2 q^{2} a b t+q^{4} a^{2} b^{2}\right)\right. \\
& \left.+q^{2}\left(1-2 p^{2} a b t+p^{4} a^{2} b^{2}\right)\right\}\left(a^{2}-2 a b t+b^{2}\right)^{2} \\
& -(p+q)^{2}\left\{1-\frac{1}{2}(p+q)^{2} a b t+\frac{1}{16}(p+q)^{4} a^{2} b^{2}\right\} \\
& \times\left(1-2 p^{2} a b t+p^{4} a^{2} b^{2}\right)\left(1-2 q^{2} a b t+q^{4} a^{2} b^{2}\right) \\
- & p^{2} q^{2}(p+q)^{2}\left\{1-\frac{1}{2}(p+q)^{2} a b t+\frac{1}{16}(p+q)^{4} a^{2} b^{2}\right\}\left(a^{2}-2 a b t+b^{2}\right)^{2} .
\end{aligned}
$$

It is clear that the previous formula is a polynomial whose degree is at most 3 with respect to $t$. Although the rest of the proof is just expanding the formula above, we describe a process to calculate for the convenience of readers.

$$
\begin{aligned}
\left\{p^{2} B^{2}\right. & \left.+q^{2} A^{2}\right\} C^{4} \\
= & -p^{2} q^{2}(p+q)^{4} a^{3} b^{3} t^{3} \\
& +(p+q)^{2} a^{2} b^{2}\left\{4 p^{2} q^{2}\left(1+\frac{1}{16}(p+q)^{4} a^{2} b^{2}\right)\right. \\
& \left.+\frac{1}{4}(p+q)^{2}\left(p^{2}+q^{2}+p^{2} q^{4} a^{2} b^{2}+p^{4} q^{2} a^{2} b^{2}\right)\right\} t^{2} \\
& + \text { terms of degree at most } 1 \\
\frac{1}{2}(p+ & q)^{2}\left\{p^{2} B^{2}+q^{2} A^{2}\right\} C^{2} D^{2} \\
= & -2 p^{2} q^{2}(p+q)^{4} a^{3} b^{3} t^{3} \\
& +(p+q)^{2} a^{2} b^{2}\left\{p^{2} q^{2}(p+q)^{2}\left(a^{2}+b^{2}\right)+4 p^{2} q^{2}\left(1+\frac{1}{16}(p+q)^{4} a^{2} b^{2}\right)\right. \\
& \left.+\frac{1}{2}(p+q)^{2}\left(p^{2}+q^{2}+p^{2} q^{4} a^{2} b^{2}+p^{4} q^{2} a^{2} b^{2}\right)\right\} t^{2} \\
& + \text { terms of degree at most } 1,
\end{aligned}
$$




$$
\begin{aligned}
\frac{1}{16}(p & +q)^{4}\left\{p^{2} B^{2}+q^{2} A^{2}\right\} D^{4} \\
= & -p^{2} q^{2}(p+q)^{4} a^{3} b^{3} t^{3} \\
& +(p+q)^{2} a^{2} b^{2}\left\{p^{2} q^{2}(p+q)^{2}\left(a^{2}+b^{2}\right)\right. \\
& \left.+\frac{1}{4}(p+q)^{2}\left(p^{2}+q^{2}+p^{2} q^{4} a^{2} b^{2}+p^{4} q^{2} a^{2} b^{2}\right)\right\} t^{2} \\
& + \text { terms of degree at most } 1, \\
-(p+ & +)^{2} C^{2} A^{2} B^{2} \\
= & 2 p^{2} q^{2}(p+q)^{4} a^{3} b^{3} t^{3} \\
& -(p+q)^{2} a^{2} b^{2}\left\{p^{2}(p+q)^{2}\left(1+q^{4} a^{2} b^{2}\right)+q^{2}(p+q)^{2}\left(1+p^{4} a^{2} b^{2}\right)\right. \\
& \left.+4 p^{2} q^{2}\left(1+\frac{1}{16}(p+q)^{4} a^{2} b^{2}\right)\right\} t^{2} \\
& + \text { terms of degree at most } 1, \\
-p^{2} & q^{2}(p+q)^{2} C^{2} D^{4} \\
= & 2 p^{2} q^{2}(p+q)^{4} a^{3} b^{3} t^{3} \\
& -(p+q)^{2} a^{2} b^{2}\left\{2 p^{2} q^{2}(p+q)^{2}\left(a^{2}+b^{2}\right)+4 p^{2} q^{2}\left(1+\frac{1}{16}(p+q)^{4} a^{2} b^{2}\right)\right\} t^{2} \\
& + \text { terms of degree at most } 1 .
\end{aligned}
$$

It is not difficult to verify that the terms of degree 3 and 2 actually cancel each other, respectively. Thus the sum of the first two terms in formula (3.3) consists of terms at most degree 1. This completes the proof of Lemma 3.2.

Lemma 3.3 The factor $C^{4}-\frac{1}{2}(p+q)^{2} C^{2} D^{2}+\frac{1}{16}(p+q)^{4} D^{4}$ in formula (3.3) does not depend on $t$.

Proof It is straightforward to see that

$$
C^{4}-\frac{1}{2}(p+q)^{2} C^{2} D^{2}+\frac{1}{16}(p+q)^{4} D^{4}=\left\{1+\frac{1}{16}(p+q)^{4} a^{2} b^{2}-\frac{1}{4}(p+q)^{2}\left(a^{2}+b^{2}\right)\right\}^{2} .
$$

This completes the proof of Lemma 3.3.

Lemma 3.4 Formula (3.3) is a concave function with respect to $t$.

Proof By Lemma 3.2 and Lemma 3.3, it is sufficient to show that $A B$ is concave with respect to $t$. It is easy to show that

$$
\begin{aligned}
\frac{d}{d t}(A B)= & -a b\left\{p^{2}\left(1-2 p^{2} a b t+p^{4} a^{2} b^{2}\right)^{-\frac{1}{2}}\left(1-2 q^{2} a b t+q^{4} a^{2} b^{2}\right)^{\frac{1}{2}}\right. \\
& \left.+q^{2}\left(1-2 p^{2} a b t+p^{4} a^{2} b^{2}\right)^{\frac{1}{2}}\left(1-2 q^{2} a b t+q^{4} a^{2} b^{2}\right)^{-\frac{1}{2}}\right\}
\end{aligned}
$$




$$
\begin{aligned}
\frac{d^{2}}{d t^{2}}(A B)= & -a^{2} b^{2}\left(1-2 p^{2} a b t+p^{4} a^{2} b^{2}\right)^{-\frac{1}{2}}\left(1-2 q^{2} a b t+q^{4} a^{2} b^{2}\right)^{-\frac{1}{2}} \\
& \times\left\{p^{2}\left(1-2 p^{2} a b t+p^{4} a^{2} b^{2}\right)^{-\frac{1}{2}}\left(1-2 q^{2} a b t+q^{4} a^{2} b^{2}\right)^{\frac{1}{2}}\right. \\
& \left.-q^{2}\left(1-2 p^{2} a b t+p^{4} a^{2} b^{2}\right)^{\frac{1}{2}}\left(1-2 q^{2} a b t+q^{4} a^{2} b^{2}\right)^{-\frac{1}{2}}\right\}^{2} \leq 0 .
\end{aligned}
$$

This completes the proof of Lemma 3.4.

Considering formula (3.3) as a function of $-1 \leq t \leq 1$, it takes the minimum value at -1 or 1 by Lemma 3.4. Therefore, our proof of Theorem 3.1 can be reduced to showing the next lemma.

Lemma 3.5 Theorem 3.1 holds for real numbers $\alpha, \beta$.

Proof In the case where $\alpha$ and $\beta$ are real numbers, we can show (3.2) directly. Since $p(1-$ $\left.q^{2} \alpha \beta\right)+q\left(1-p^{2} \alpha \beta\right)=(p+q)(1-p q \alpha \beta)$, it is sufficient to show

$$
\begin{aligned}
& (1-p q \alpha \beta)\left\{\left(1-\frac{1}{4}(p+q)^{2} \alpha \beta\right)^{2}+\frac{1}{4}(p+q)^{2}(\alpha-\beta)^{2}\right\} \\
& -\left(1-\frac{1}{4}(p+q)^{2} \alpha \beta\right)\left\{\left(1-p^{2} \alpha \beta\right)\left(1-q^{2} \alpha \beta\right)+p q(\alpha-\beta)^{2}\right\} \geq 0 .
\end{aligned}
$$

It is easy to calculate the left-hand side of the previous formula as follows:

$$
\begin{aligned}
= & (1-p q \alpha \beta)\left\{1-\frac{1}{2}(p+q)^{2} \alpha \beta+\frac{1}{16}(p+q)^{4} \alpha^{2} \beta^{2}+\frac{1}{4}(p+q)^{2}\left(\alpha^{2}-2 \alpha \beta+\beta^{2}\right)\right\} \\
& -\left(1-\frac{1}{4}(p+q)^{2} \alpha \beta\right)\left\{1-p^{2} \alpha \beta-q^{2} \alpha \beta+p^{2} q^{2} \alpha^{2} \beta^{2}+p q\left(\alpha^{2}-2 \alpha \beta+\beta^{2}\right)\right\} \\
= & 1-\frac{1}{2}(p+q)^{2} \alpha \beta+\frac{1}{16}(p+q)^{4} \alpha^{2} \beta^{2}+\frac{1}{4}(p+q)^{2}\left(\alpha^{2}-2 \alpha \beta+\beta^{2}\right) \\
& -p q \alpha \beta+\frac{1}{2} p q(p+q)^{2} \alpha^{2} \beta^{2}-\frac{1}{16} p q(p+q)^{4} \alpha^{3} \beta^{3} \\
& -\frac{1}{4} p q(p+q)^{2}\left(\alpha^{3} \beta-2 \alpha^{2} \beta^{2}+\alpha \beta^{3}\right) \\
& -1+p^{2} \alpha \beta+q^{2} \alpha \beta-p^{2} q^{2} \alpha^{2} \beta^{2}-p q\left(\alpha^{2}-2 \alpha \beta+\beta^{2}\right) \\
& +\frac{1}{4}(p+q)^{2} \alpha \beta-\frac{1}{4} p^{2}(p+q)^{2} \alpha^{2} \beta^{2}-\frac{1}{4} q^{2}(p+q)^{2} \alpha^{2} \beta^{2}+\frac{1}{4} p^{2} q^{2}(p+q)^{2} \alpha^{3} \beta^{3} \\
& +\frac{1}{4} p q(p+q)^{2}\left(\alpha^{3} \beta-2 \alpha^{2} \beta^{2}+\alpha \beta^{3}\right) \\
= & \frac{(p-q)^{2}}{16}\left\{4\left(\alpha^{2}+\alpha \beta+\beta^{2}\right)-\left((p+q)^{2}+2\left(p^{2}+q^{2}\right)\right) \alpha^{2} \beta^{2}-p q(p+q)^{2} \alpha^{3} \beta^{3}\right\} .
\end{aligned}
$$

Thus, we need to show, for any $0 \leq p, q \leq 1,-1 \leq \alpha, \beta \leq 1$,

$$
4\left(\alpha^{2}+\alpha \beta+\beta^{2}\right)-\left((p+q)^{2}+2\left(p^{2}+q^{2}\right)\right) \alpha^{2} \beta^{2}-p q(p+q)^{2} \alpha^{3} \beta^{3} \geq 0 .
$$


(i) The case where $\alpha \beta \geq 0$.

$$
\begin{aligned}
& 4\left(\alpha^{2}+\alpha \beta+\beta^{2}\right)-\left((p+q)^{2}+2\left(p^{2}+q^{2}\right)\right) \alpha^{2} \beta^{2}-p q(p+q)^{2} \alpha^{3} \beta^{3} \\
& \quad \geq 4\left(\alpha^{2}+\alpha \beta+\beta^{2}\right)-8 \alpha^{2} \beta^{2}-4 \alpha^{3} \beta^{3} \\
& \quad \geq 4\left(\alpha^{2}+\alpha \beta+\beta^{2}\right)-8 \alpha \beta-4 \alpha \beta=4(\alpha-\beta)^{2} \geq 0 .
\end{aligned}
$$

(ii) The case where $\alpha \beta<0$.

$$
\begin{aligned}
& 4\left(\alpha^{2}+\alpha \beta+\beta^{2}\right)-\left((p+q)^{2}+2\left(p^{2}+q^{2}\right)\right) \alpha^{2} \beta^{2}-p q(p+q)^{2} \alpha^{3} \beta^{3} \\
& \quad=4(\alpha+\beta)^{2}-\alpha \beta\left\{4+\left((p+q)^{2}+2\left(p^{2}+q^{2}\right)\right) \alpha \beta+p q(p+q)^{2} \alpha^{2} \beta^{2}\right\} \geq 0,
\end{aligned}
$$

where we used $\alpha \beta<0$ and the fact

$$
4+\left((p+q)^{2}+2\left(p^{2}+q^{2}\right)\right) \alpha \beta+p q(p+q)^{2} \alpha^{2} \beta^{2} \geq 0 .
$$

Indeed, put $x=\alpha \beta$,

$$
f(x)=p q(p+q)^{2} x^{2}+\left((p+q)^{2}+2\left(p^{2}+q^{2}\right)\right) x+4
$$

and consider the minimum value of $f(x)$ for $-1 \leq x \leq 0$. It is obvious

$$
f^{\prime}(x)=0 \quad \text { if and only if } \quad x=-\frac{(p+q)^{2}+2\left(p^{2}+q^{2}\right)}{2 p q(p+q)^{2}} \leq-1,
$$

because $p q(p+q)^{2} \leq(p+q)^{2} \leq 2\left(p^{2}+q^{2}\right)$. Thus the minimum value of $f(x)$ for $-1 \leq x \leq 0$ is $f(-1)$. Now it is immediate to see

$$
f(-1)=2\left(1-p^{2}\right)\left(1-q^{2}\right)+(1-p q)\left(2-p^{2}-q^{2}\right) \geq 0 .
$$

It is easy to see the equality condition. This completes the proof of Lemma 3.5.

Completion of the proof of Theorem 3.1 Thus we have shown that formula (3.3) is nonnegative. It is easy to check that the equality condition of Theorem 3.1 holds, because it can be reduced to the equality condition for the case of real numbers. This completes the proof of Theorem 3.1.

The following theorem can be regarded as the most natural extension of the CBS type inequality in complex inner product spaces in the context of relation between inner product spaces and the Möbius operations. In the rest of the paper, we simply denote $\oplus_{1}, \otimes_{1}$ by $\oplus, \otimes$, respectively.

Theorem 3.6 Let $\mathbb{V}$ be a complex inner product space. For any $u, v \in \mathbb{V}, s>\max \{\|u\|,\|v\|\}$, and $w \in \mathbb{V}$ with $\|w\| \leq 1$, the following inequality holds:

$$
\left|\frac{\langle u, w\rangle-\langle v, w\rangle}{1-\frac{1}{s^{2}} \overline{\langle u, w\rangle}\langle v, w\rangle}\right| \leq\|w\| \otimes_{s} \sqrt{\frac{\|u\|^{2}-2 \operatorname{Re}\langle u, v\rangle+\|v\|^{2}}{1-\frac{2}{s^{2}} \operatorname{Re}\langle u, v\rangle+\frac{1}{s^{4}}\|u\|^{2}\|v\|^{2}}},
$$


that is,

$$
\left|\frac{\langle u, w\rangle-\langle v, w\rangle}{1-\frac{1}{s^{2}} \overline{\langle u, w\rangle}\langle v, w\rangle}\right| \leq s \tanh \left\{\|w\| \tanh ^{-1}\left(\frac{1}{s} \sqrt{\frac{\|u\|^{2}-2 \operatorname{Re}\langle u, v\rangle+\|v\|^{2}}{1-\frac{2}{s^{2}} \operatorname{Re}\langle u, v\rangle+\frac{1}{s^{4}}\|u\|^{2}\|v\|^{2}}}\right)\right\} .
$$

The equality holds if and only if one of the following conditions is satisfied:

(i) $u=v$,

(ii) $w=0$,

(iii) $\|w\|=1$ and $u=\lambda w, v=\mu w$ for some complex numbers $\lambda$, $\mu$.

Proof At first, we show that if $\|u\|,\|v\|<1$ and $\|w\| \leq 1$, then

$$
\left|\frac{\langle u, w\rangle-\langle v, w\rangle}{1-\overline{\langle u, w\rangle}\langle v, w\rangle}\right| \leq\|w\| \otimes \sqrt{\frac{\|u\|^{2}-2 \operatorname{Re}\langle u, v\rangle+\|v\|^{2}}{1-2 \operatorname{Re}\langle u, v\rangle+\|u\|^{2}\|v\|^{2}}} .
$$

The proof uses an argument in [2] that shows the operator monotonicity of the functions $t^{p}$ with $0 \leq p \leq 1$. Let $E$ denote the set of all real numbers $p$ in the interval $[0,1]$ for which if $w \in \mathbb{V}$ with $\|w\|=p$ then inequality (3.5) holds. Trivially $0 \in E$, and Theorem 1.7 implies that $1 \in E$. It is obvious that $E$ is a closed subset of $[0,1]$. The proof of inequality (3.5) will be complete when we show that $E$ is convex. Suppose $p, q \in E$. For an arbitrary element $w \in \mathbb{V}$ with $\|w\|=\frac{p+q}{2}$, put $w_{1}=\frac{2 p}{p+q} w, w_{2}=\frac{2 q}{p+q} w$. Then $\left\|w_{1}\right\|=p,\left\|w_{2}\right\|=q$, so we have

$$
\begin{aligned}
& \left|\frac{\left\langle u, w_{1}\right\rangle-\left\langle v, w_{1}\right\rangle}{1-\overline{\left\langle u, w_{1}\right\rangle}\left\langle v, w_{1}\right\rangle}\right| \leq p \otimes \sqrt{\frac{\|u\|^{2}-2 \operatorname{Re}\langle u, v\rangle+\|v\|^{2}}{1-2 \operatorname{Re}\langle u, v\rangle+\|u\|^{2}\|v\|^{2}}}, \\
& \left|\frac{\left\langle u, w_{2}\right\rangle-\left\langle v, w_{2}\right\rangle}{1-\overline{\left\langle u, w_{2}\right\rangle}\left\langle v, w_{2}\right\rangle}\right| \leq q \otimes \sqrt{\frac{\|u\|^{2}-2 \operatorname{Re}\langle u, v\rangle+\|v\|^{2}}{1-2 \operatorname{Re}\langle u, v\rangle+\|u\|^{2}\|v\|^{2}}} .
\end{aligned}
$$

We have to show

$$
\left|\frac{\langle u, w\rangle-\langle v, w\rangle}{1-\overline{\langle u, w\rangle}\langle v, w\rangle}\right| \leq \frac{p+q}{2} \otimes \sqrt{\frac{\|u\|^{2}-2 \operatorname{Re}\langle u, v\rangle+\|v\|^{2}}{1-2 \operatorname{Re}\langle u, v\rangle+\|u\|^{2}\|v\|^{2}}} .
$$

By the properties of the Möbius operations together with ordinary addition and multiplication restricted to real numbers and Lemma 1.5,

$$
\begin{aligned}
\frac{p+q}{2} \otimes \sqrt{\frac{\|u\|^{2}-2 \operatorname{Re}\langle u, v\rangle+\|v\|^{2}}{1-2 \operatorname{Re}\langle u, v\rangle+\|u\|^{2}\|v\|^{2}}} \\
=\frac{1}{2} \otimes\left(p \otimes \sqrt{\frac{\|u\|^{2}-2 \operatorname{Re}\langle u, v\rangle+\|v\|^{2}}{1-2 \operatorname{Re}\langle u, v\rangle+\|u\|^{2}\|v\|^{2}}}\right) \oplus \frac{1}{2} \\
\otimes\left(q \otimes \sqrt{\frac{\|u\|^{2}-2 \operatorname{Re}\langle u, v\rangle+\|v\|^{2}}{1-2 \operatorname{Re}\langle u, v\rangle+\|u\|^{2}\|v\|^{2}}}\right) \\
\geq \frac{1}{2} \otimes\left|\frac{\left\langle u, w_{1}\right\rangle-\left\langle v, w_{1}\right\rangle}{1-\overline{\left\langle u, w_{1}\right\rangle}\left\langle v, w_{1}\right\rangle}\right| \oplus \frac{1}{2} \otimes\left|\frac{\left\langle u, w_{2}\right\rangle-\left\langle v, w_{2}\right\rangle}{1-\overline{\left\langle u, w_{2}\right\rangle}\left\langle v, w_{2}\right\rangle}\right| .
\end{aligned}
$$


It is also clear that

$$
c \leq \frac{1}{2} \otimes b_{1} \oplus \frac{1}{2} \otimes b_{2}
$$

is equivalent to

$$
2 \otimes c \leq b_{1} \oplus b_{2}
$$

Therefore, in order to show (3.6), it is sufficient to prove

$$
2 \otimes\left|\frac{\langle u, w\rangle-\langle v, w\rangle}{1-\overline{\langle u, w\rangle}\langle v, w\rangle}\right| \leq\left|\frac{\left\langle u, w_{1}\right\rangle-\left\langle v, w_{1}\right\rangle}{1-\overline{\left\langle u, w_{1}\right\rangle}\left\langle v, w_{1}\right\rangle}\right| \oplus\left|\frac{\left\langle u, w_{2}\right\rangle-\left\langle v, w_{2}\right\rangle}{1-\overline{\left\langle u, w_{2}\right\rangle}\left\langle v, w_{2}\right\rangle}\right| .
$$

We put $\alpha=\frac{2}{p+q}\langle u, w\rangle, \beta=\frac{2}{p+q}\langle v, w\rangle$. Then it follows that $|\alpha|,|\beta|<1$ by the classical CBS inequality. Furthermore, we have

$$
\begin{aligned}
& \langle u, w\rangle=\frac{p+q}{2} \alpha, \quad\langle v, w\rangle=\frac{p+q}{2} \beta, \quad\left\langle u, w_{1}\right\rangle=p \alpha, \quad\left\langle v, w_{1}\right\rangle=p \beta, \\
& \left\langle u, w_{2}\right\rangle=q \alpha, \quad\left\langle v, w_{2}\right\rangle=q \beta .
\end{aligned}
$$

Hence we can obtain

$$
\begin{aligned}
& \left|\frac{\left\langle u, w_{1}\right\rangle-\left\langle v, w_{1}\right\rangle}{1-\overline{\left\langle u, w_{1}\right\rangle}\left\langle v, w_{1}\right\rangle}\right| \oplus\left|\frac{\left\langle u, w_{2}\right\rangle-\left\langle v, w_{2}\right\rangle}{1-\overline{\left\langle u, w_{2}\right\rangle}\left\langle v, w_{2}\right\rangle}\right|=\frac{\left.\left|\frac{\left\langle u, w_{1}\right\rangle-\left\langle v, w_{1}\right\rangle}{1-\left\langle u, w_{1}\right\rangle\left\langle v, w_{1}\right\rangle}\right|+\left|\frac{\left\langle u, w_{2}\right\rangle-\left\langle v, w_{2}\right\rangle}{1-\left\langle u, w_{2}\right\rangle}\right| v, w_{2}\right\rangle}{1+\left|\frac{\left\langle u, w_{1}\right\rangle-\left\langle v, w_{1}\right\rangle}{1-\overline{\left\langle u, w_{1}\right\rangle}\left\langle v, w_{1}\right\rangle}\right| \frac{\left\langle u, w_{2}\right\rangle-\left\langle v, w_{2}\right\rangle}{1-\overline{\left\langle u, w_{2}\right\rangle}\left\langle v, w_{2}\right\rangle} \mid} \\
& =\frac{\left|1-q^{2} \bar{\alpha} \beta\right| p|\alpha-\beta|+\left|1-p^{2} \bar{\alpha} \beta\right| q|\alpha-\beta|}{\left|1-p^{2} \bar{\alpha} \beta\right|\left|1-q^{2} \bar{\alpha} \beta\right|+p q|\alpha-\beta|^{2}}
\end{aligned}
$$

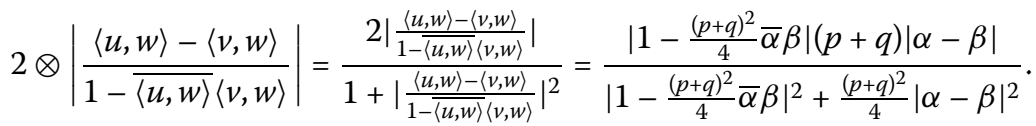

Therefore, dividing both sides by $|\alpha-\beta|$, our proof is reduced to show that

$$
\frac{\left|1-\frac{(p+q)^{2}}{4} \bar{\alpha} \beta\right|(p+q)}{\left|1-\frac{(p+q)^{2}}{4} \bar{\alpha} \beta\right|^{2}+\frac{(p+q)^{2}}{4}|\alpha-\beta|^{2}} \leq \frac{\left|1-q^{2} \bar{\alpha} \beta\right| p+\left|1-p^{2} \bar{\alpha} \beta\right| q}{\left|1-p^{2} \bar{\alpha} \beta\right|\left|1-q^{2} \bar{\alpha} \beta\right|+p q|\alpha-\beta|^{2}}
$$

the last inequality actually holds by Theorem 3.1. Thus the set $E$ is convex, which leads to $E=[0,1]$, so inequality $(3.5)$ holds.

Finally, let $u, v \in \mathbb{V}$ be arbitrary elements, and let $s>\max \{\|u\|,\|v\|\}$. Applying inequality (3.5) to $\frac{u}{s}, \frac{v}{s}$, it is straightforward to deduce inequality (3.4).

For the equality condition, we may assume $s=1$.

It is immediate to see that the equality in (3.5) holds provided one of the conditions (i), (ii), or (iii) is satisfied.

Conversely, suppose $\frac{1}{2} \leq p<1, u, v, w \in \mathbb{V}$ with $\|u\|<1,\|v\|<1,\|w\|=p$ and that the equality of (3.5) holds. If we put

$$
p^{\prime}=2 p-1, \quad w_{1}=\frac{2 p^{\prime}}{p^{\prime}+1} w, \quad w_{2}=\frac{2}{p^{\prime}+1} w
$$


then it is obvious that $\left\|w_{1}\right\|=p^{\prime},\left\|w_{2}\right\|=1$. By Theorem 3.1, inequality (3.5) and Lemma 1.5, it follows that

$$
\begin{aligned}
\left|\frac{\langle u, w\rangle-\langle v, w\rangle}{1-\overline{\langle u, w\rangle}\langle v, w\rangle}\right| \leq & \frac{1}{2} \otimes\left|\frac{\left\langle u, w_{1}\right\rangle-\left\langle v, w_{1}\right\rangle}{1-\overline{\left\langle u, w_{1}\right\rangle}\left\langle v, w_{1}\right\rangle}\right| \oplus \frac{1}{2} \otimes\left|\frac{\left\langle u, w_{2}\right\rangle-\left\langle v, w_{2}\right\rangle}{1-\overline{\left\langle u, w_{2}\right\rangle}\left\langle v, w_{2}\right\rangle}\right| \\
\leq & \frac{1}{2} \otimes\left(p^{\prime} \otimes \sqrt{\frac{\|u\|^{2}-2 \operatorname{Re}\langle u, v\rangle+\|v\|^{2}}{1-2 \operatorname{Re}\langle u, v\rangle+\|u\|^{2}\|v\|^{2}}}\right) \oplus \frac{1}{2} \\
& \otimes\left(1 \otimes \sqrt{\frac{\|u\|^{2}-2 \operatorname{Re}\langle u, v\rangle+\|v\|^{2}}{1-2 \operatorname{Re}\langle u, v\rangle+\|u\|^{2}\|v\|^{2}}}\right) \\
= & \frac{p^{\prime}+1}{2} \otimes \sqrt{\frac{\|u\|^{2}-2 \operatorname{Re}\langle u, v\rangle+\|v\|^{2}}{1-2 \operatorname{Re}\langle u, v\rangle+\|u\|^{2}\|v\|^{2}}},
\end{aligned}
$$

where the last formula equals to the first one by the assumption. Therefore, by the equality condition of Lemma 1.5,

$$
\left|\frac{\left\langle u, w_{2}\right\rangle-\left\langle v, w_{2}\right\rangle}{1-\overline{\left\langle u, w_{2}\right\rangle}\left\langle v, w_{2}\right\rangle}\right|=\sqrt{\frac{\|u\|^{2}-2 \operatorname{Re}\langle u, v\rangle+\|v\|^{2}}{1-2 \operatorname{Re}\langle u, v\rangle+\|u\|^{2}\|v\|^{2}}},
$$

which yields one of the following conditions:

(i) $u=v$,

(ii) $u=\lambda w, v=\mu w$ for some complex numbers $\lambda, \mu$

by the equality condition of Theorem 1.7. We have to consider only the case of condition (ii). The first inequality in the previous series of inequalities also has to be an equality. Namely,

$$
\left|\frac{\langle u, w\rangle-\langle v, w\rangle}{1-\overline{\langle u, w\rangle}\langle v, w\rangle}\right|=\frac{1}{2} \otimes\left|\frac{\left\langle u, w_{1}\right\rangle-\left\langle v, w_{1}\right\rangle}{1-\overline{\left\langle u, w_{1}\right\rangle}\left\langle v, w_{1}\right\rangle}\right| \oplus \frac{1}{2} \otimes\left|\frac{\left\langle u, w_{2}\right\rangle-\left\langle v, w_{2}\right\rangle}{1-\overline{\left\langle u, w_{2}\right\rangle}\left\langle v, w_{2}\right\rangle}\right|,
$$

which yields one of the following conditions:

(i') $p^{\prime}=1$,

(ii') $\alpha=\beta$.

However, we have $p^{\prime} \neq 1$, because $p<1$. So the condition (ii') $\alpha=\beta$ has to occur. Furthermore,

$$
\alpha=\frac{2}{p^{\prime}+1}\langle u, w\rangle=\frac{2}{p^{\prime}+1} \lambda\|w\|^{2} \quad \text { and } \quad \beta=\frac{2}{p^{\prime}+1}\langle v, w\rangle=\frac{2}{p^{\prime}+1} \mu\|w\|^{2},
$$

thus we can conclude $\lambda=\mu$ or $u=v$.

Next, suppose $\frac{1}{4} \leq p<\frac{1}{2}$ and that the equality of (3.5) holds for $\|w\|=p$. Put

$$
p^{\prime}=2 p-\frac{1}{2}, \quad w_{1}=\frac{2 p^{\prime}}{p^{\prime}+\frac{1}{2}} w, \quad w_{2}=\frac{2 \cdot \frac{1}{2}}{p^{\prime}+\frac{1}{2}} w .
$$

Then we have $\left\|w_{1}\right\|=p^{\prime},\left\|w_{2}\right\|=\frac{1}{2}$. A similar argument before shows

$$
\left|\frac{\left\langle u, w_{2}\right\rangle-\left\langle v, w_{2}\right\rangle}{1-\overline{\left\langle u, w_{2}\right\rangle}\left\langle v, w_{2}\right\rangle}\right|=\frac{1}{2} \otimes \sqrt{\frac{\|u\|^{2}-2 \operatorname{Re}\langle u, v\rangle+\|v\|^{2}}{1-2 \operatorname{Re}\langle u, v\rangle+\|u\|^{2}\|v\|^{2}}},
$$


which yields $u=v$ by the equality condition established just before. This argument can be repeated for half open intervals $\left[1 / 2^{n+1}, 1 / 2^{n}\right)$, and we can conclude that the equality condition holds. This completes the proof.

The following theorem shows that the number $\|w\|$ in the right-hand side of inequality (3.4) is the best in a sense.

Theorem 3.7 Let $\mathbb{V}$ be a complex inner product space and $w$ be a fixed element in $\mathbb{V}$ with $\|w\| \leq 1$. Suppose that there exists a nonnegative constant $K$ satisfying the following inequality:

$$
\left|\frac{\langle u, w\rangle-\langle v, w\rangle}{1-\overline{\langle u, w\rangle}\langle v, w\rangle}\right| \leq K \otimes \sqrt{\frac{\|u\|^{2}-2 \operatorname{Re}\langle u, v\rangle+\|v\|^{2}}{1-2 \operatorname{Re}\langle u, v\rangle+\|u\|^{2}\|v\|^{2}}}
$$

for any $u, v \in \mathbb{V}$ with $\|u\|,\|v\|<1$. Then $\|w\| \leq K$.

Proof For any $u, v \in \mathbb{V}$ and $s>\max \{\|u\|,\|v\|\}$, by applying assumption (3.7) to $\frac{u}{s}, \frac{v}{s}$, it is immediate to see

$$
\left|\frac{\langle u, w\rangle-\langle v, w\rangle}{1-\frac{1}{s^{2}} \overline{\langle u, w\rangle}\langle v, w\rangle}\right| \leq s \tanh \left\{K \tanh ^{-1}\left(\frac{1}{s} \sqrt{\frac{\|u\|^{2}-2 \operatorname{Re}\langle u, v\rangle+\|v\|^{2}}{1-\frac{2}{s^{2}} \operatorname{Re}\langle u, v\rangle+\frac{1}{s^{4}}\|u\|^{2}\|v\|^{2}}}\right)\right\} .
$$

By letting $s \rightarrow \infty$, we can obtain

$$
|\langle u, w\rangle-\langle v, w\rangle| \leq K\|u-v\| .
$$

If we take $u=\frac{1}{2} w$ and $v=0$, then we have

$$
\frac{1}{2}\|w\|^{2}=|\langle u, w\rangle-\langle v, w\rangle| \leq K\|u-v\|=\frac{K}{2}\|w\|,
$$

which yields $\|w\| \leq K$. This completes the proof.

Lemma 3.5 implies the result corresponding to Theorem 3.6 in real inner product spaces.

Theorem 3.8 Let $\mathbb{V}$ be a real inner product space. For any $u, v \in \mathbb{V}, s>\max \{\|u\|,\|v\|\}$, and $w \in \mathbb{V}$ with $\|w\| \leq 1$, the following inequality holds:

$$
\left|\langle u, w\rangle \ominus_{s}\langle v, w\rangle\right| \leq\|w\| \otimes_{s}\left\|u \ominus_{s} v\right\|
$$

or

$$
h(\langle u, w\rangle,\langle v, w\rangle) \leq\|w\| h(u, v)
$$

that is,

$$
\left|\frac{\langle u, w\rangle-\langle v, w\rangle}{1-\frac{1}{s^{2}}\langle u, w\rangle\langle v, w\rangle}\right| \leq s \tanh \left\{\|w\| \tanh ^{-1}\left(\frac{1}{s} \sqrt{\frac{\|u\|^{2}-2\langle u, v\rangle+\|v\|^{2}}{1-\frac{2}{s^{2}}\langle u, v\rangle+\frac{1}{s^{4}}\|u\|^{2}\|v\|^{2}}}\right)\right\} .
$$

The equality holds if and only if one of the following conditions is satisfied: 
(i) $u=v$,

(ii) $w=0$,

(iii) $\|w\|=1$ and $u=\lambda w, v=\mu w$ for some real numbers $\lambda, \mu$.

\section{Remark 3.9}

(i) Obviously, if $\|w\|=1$, then inequalities (2.1) and (3.4) reduce to inequality (1.1).

(ii) As used in the proof of Theorem 3.7, by letting $s \rightarrow \infty$ in inequality (3.4), we can recapture the classical CBS inequality (1.2).

(iii) Theorem 3.6 is an improvement of Theorem 1.7, because, if $0 \leq a<s$ and $\|w\| \leq 1$, then $\|w\| \otimes_{s} a \leq a$.

\section{Acknowledgements}

The author is greatly indebted to the referees who read the manuscript carefully, as their comments improved presentation of the paper. Especially, one of the referees remarkably simplified the proof of Theorem 2.2. The author would like to thank Prof. Sin-Ei Takahasi for raising Question 1.10 in an oral presentation by the author at the conference 92nd Yonezawa Mathematics Seminar on June 30th, 2018. This work was supported by the Research Institute for Mathematical Sciences, a Joint Usage/Research Center located in Kyoto University.

\section{Funding}

Not applicable.

\section{Availability of data and materials}

Not applicable.

\section{Competing interests}

The author declares that he has no competing interests.

\section{Authors' contributions}

The author read and approved the final manuscript.

\section{Publisher's Note}

Springer Nature remains neutral with regard to jurisdictional claims in published maps and institutional affiliations.

Received: 13 December 2018 Accepted: 10 June 2019 Published online: 24 June 2019

\section{References}

1. Conway, J.B.: A Course in Functional Analysis, 2nd edn. Graduate Texts in Mathematics, vol. 96. Springer, New York (1990)

2. Pedersen, G.K.: Some operator monotone functions. Proc. Am. Math. Soc. 36(1), 309-310 (1972)

3. Steele, J.M.: The Cauchy-Schwarz Master Class: An Introduction to the Art of Mathematical Inequalities. MAA Problem Books Series. Cambridge University Press, Cambridge (2008)

4. Ungar, A.A.: Thomas rotation and the parametrization of the Lorentz transformation group. Found. Phys. Lett. 1(1), 57-89 (1988)

5. Ungar, A.A.: Group-like structure underlying the unit ball in real inner product spaces. Results Math. 18(3-4), 355-364 (1990)

6. Ungar, A.A.: Extension of the unit disk gyrogroup into the unit ball of any real inner product space. J. Math. Anal. Appl. 202(3), 1040-1057 (1996)

7. Ungar, A.A.: Analytic Hyperbolic Geometry and Albert Einstein's Special Theory of Relativity. World Scientific, Singapore (2008)

8. Watanabe, K.: A Cauchy type inequality for Möbius operations. J. Inequal. Appl. 2018, 97 (2018). https://doi.org/10.1186/s13660-018-1690-2

9. Watanabe, K.: A Cauchy-Bunyakovsky-Schwarz type inequality related to the Möbius addition. J. Math. Inequal. 12(4), 989-996 (2018). https://doi.org/10.7153/jmi-2018-12-75

10. Watanabe, K.: Orthogonal gyroexpansion in Möbius gyrovector spaces. J. Funct. Spaces 2017, Article ID 1518254 (2017). https://doi.org/10.1155/2017/1518254 